\title{
Nuclear EpICD expression and its role in hepatocellular carcinoma
}

\author{
SHIN YOUNG PARK ${ }^{1}$, JUN SANG BAE ${ }^{2}$, EUN JUNG CHA ${ }^{1}$, HYUN HEE CHU ${ }^{1}$, \\ JANG SIHN SOHN $^{1}$ and WOO SUNG MOON ${ }^{2}$
}

\author{
${ }^{1}$ Department of Pathology, Konyang University College of Medicine, Daejeon 35365; ${ }^{2}$ Department of Pathology, \\ Chonbuk National University, Medical School Research Institute of Clinical Medicine of \\ Chonbuk National University-Biomedical Research Institute of Chonbuk National University Hospital \\ and Research Institute for Endocrine Sciences, Jeonju 561-756, Republic of Korea
}

Received January 13, 2016; Accepted February 18, 2016

DOI: $10.3892 /$ or.2016.4789

\begin{abstract}
Regulated intramembrane proteolysis of epithelial cell adhesion molecule (EpCAM) results in shedding of the extracellular domain (EpEX) and release of the intracellular domain (EpICD) into the cytoplasm. Released EpICD associates with FHL2, $\beta$-catenin and Lef-1 to form a nuclear complex and triggers oncogenic signaling. This study was conducted to examine the nuclear expression of EpICD in hepatocellular carcinoma (HCC) and to assess the role of EpICD in HCC. EpICD immunoexpression was examined in 100 cases of HCC using tissue microarrays and correlated with clinicopathological parameters. We also examined the role of EpICD in HCC using EpICD cDNA transfected HCC cell line and EpCAM silenced HCC cell line by small interfering RNA (siRNA). Nuclear expression of EpICD was observed in 19 of $100(19 \%)$ cases. Nuclear expression of EpICD significantly correlated with nuclear expression of $\beta$-catenin, and Ki-67 labeling index. In addition, nuclear expression of EpICD was associated with higher histologic grade and advanced T category. Forced overexpression of EpICD in the HCC cell significantly increased the cell proliferation, migration and invasion. The overexpression of EpICD also increased the expression levels of the active form of $\beta$-catenin and c-myc and cyclin D1. In contrast, downregulation of EpCAM by siRNA decreased the cell proliferation, migration, invasion and the expression of active form of $\beta$-catenin, $c$-myc and cyclin D1. Our present data suggest that EpICD plays important roles in $\mathrm{HCC}$ progression by modulating expression of target genes of EpCAM.
\end{abstract}

Correspondence to: Professor Woo Sung Moon, Department of Pathology, Chonbuk National University, Medical School, 567 Baekje-daero, Doekjin-gu, Jeonju-si, Jeollabuk-do 561-756, Republic of Korea

E-mail: mws@chonbuk.ac.kr

Key words: carcinoma, hepatocellular, epithelial cell adhesion molecule, $\beta$-catenin

\section{Introduction}

Hepatocellular carcinoma (HCC) is the sixth most common cancer and the second leading cause of cancer-related death worldwide (1). Even though the clinical diagnosis and management of HCC have improved significantly during the last few decades, HCC is still associated with a poor prognosis (2). Therefore, considering the current limited therapy options for $\mathrm{HCC}$, finding a new therapeutic target molecule has become very important.

Epithelial cell adhesion molecule (EpCAM) is a transmembrane glycoprotein that functions as a hemophilic, epithelial-specific intercellular cell-adhesion molecule (3). EpCAM consists of an extracellular domain (EpEX), a single transmembrane domain, and a short 26-amino acid intracellular domain (EpICD) (4). EpCAM is expressed in several human epithelial tissues and cancers, including HCC and progenitor and stem cells $(4,5)$. The presence of a high amount of membranous EpCAM in various cancers has rendered EpCAM an ideal target for immunotherapy $(4,6)$. However, despite the broad distribution of EpCAM in human malignancies, results from recent clinical trials of EpCAM-specific monoclonal antibodies have shown limited efficacy $(7,8)$.

Maetzel et al reported that regulated intramembrane proteolysis (RIP)-mediated loss of EpCAM from the tumor cell surface might be one of the reasons for the limited efficacy of EpCAM-based cancer therapies (9). The cleavage of the EpCAM ectodomain, EpEx, by protease tumor necrosis factor $\alpha$ converting enzyme (TACE) and presenilin-2 (PS-2) and its shedding have been shown to release its intracellular domain (EpICD), which then translocates to the nucleus and results in the activation of oncogenic signaling (9). The association of EpICD with the FHL2 and Wnt pathway components $\beta$-catenin and Lef-1 forms a nuclear complex that binds DNA at Lef-1 consensus sites and induces gene transcription, leading to increased cell proliferation (9).

EpICD is frequently detected in various cancers, including breast, prostate, colon, bladder, thyroid, and ovarian (10,11). A recent study revealed that the nuclear expression of EpICD is correlated with cell growth and proliferation via RIP-mediated cell signaling in extrahepatic cholangiocarcinoma (12). Nuclear 
expression of EpICD is also associated with a poor prognosis in thyroid and breast cancers $(10,13)$. However, RIP-mediated cell signaling of EpCAM in HCC has not yet been studied.

Given the above background, we conducted this study to evaluate the nuclear EpICD expression in HCC and clarify the role of EpICD in the progression and prognosis of HCC.

\section{Materials and methods}

Patients and tissue samples. Formalin-fixed, paraffinembedded HCC blocks were retrieved from the archive maintained at the Department of Pathology at Chonbuk National University Hospital and also at Konyang University Hospital from 2002-2009. A total of 100 patients who underwent surgical resection were eligible, according to the following criteria: availability of hematoxylin and eosinstained glass slides and paraffin blocks for construction of a tissue microarray, no preoperative treatment, such as transarterial chemoembolization or radiation.

The clinical and pathological characteristics of the patients regarding age, gender, tumor size, histologic differentiation according to the Edmonson-Steiner grade, multiplicity, underlying etiology, presence of vascular invasion, and recurrence were obtained by a review of medical records. Patients were 36-75 years of age (mean age: 56 ) and consisted of 80 males and 20 females. Sixty-five cases were associated with hepatitis B, 4 were hepatitis C-related, 14 were alcohol related, and 18 had an unknown etiology. Overall survival was calculated from the date of surgery to the date of death or last follow-up visit. The follow-up period ranged from one to 134 months (median, 68 months). This study was approved by the Institutional Review Board of Konyang University Hospital (KYUH 2015-05-011).

Tissue microarray and immunohistochemical analysis. Tissue microarrays were constructed for immunohistochemical staining. At least two tissue cores ( $3.0 \mathrm{~mm}$ in diameter) were obtained from the most representative area in all individual cases. Additionally, normal liver tissues from each matched HCC were included as the negative controls.

Immunohistochemistry was performed on $4-\mu \mathrm{m}$ sections of tissue microarray blocks that included 100 surgically removed samples. Tissue sections were deparaffinized and rehydrated following standard procedure. Heat-induced antigen retrieval was carried out, and the sections were incubated for 30 min along with primary antibodies. Antibodies were used for EpCAM (Clone VU-1D9, Calbiochem, La Jolla, CA, USA) for the extracellular domain of EpCAM (EpEX), EpICD (Clone E144, Abcam, Cambridge, UK) and $\beta$-catenin (Clone 14, Ventana Medical Systems, Tucson, AZ, USA). Ki-67 (Clone 30-9, Ventana Medical Systems) was performed on whole tissue sections. All immunohistochemical staining was carried out using a BenchMark XT autostainer (Ventana Medical Systems).

Immunohistochemical scoring was performed by two pathologists who were blinded to the clinical outcome. For all antibodies studied except $\mathrm{Ki}-67$, the results of the immunostaining were scored based on the size of the positive area and the intensity. The proportion score was defined as follows: $0,<10 \%$ cells; $1,10-30 \%$ cells; $2,31-60 \%$ cells; $3,>60 \%$ cells. The intensity score was interpreted as follows: 0 , none; 1 , mild; 2 , moderate; 3 , strong. A total score (range: $0-6$ ) was obtained by adding the scores of proportion and intensity. EpICD, EpEX, and $\beta$-catenin positive staining was defined as a staining score $\geq 2$. Ki- 67 positivity was defined as an expression in $\geq 10 \%$ of tumor cells. The cut-off score for determining positive expression was determined by receiver-operating characteristic curve analysis.

HCC cell lines. Human HCC cell lines HLE, HLF, and Huh-7 were purchased from the Health Science Research Resources Bank (Osaka, Japan). The HepG2 cell line was obtained from the American Type Culture Collection (Manassas, VA, USA). In addition, sarcomatoid HCC cell line (SH-J1) was also used (14). All HCC cell lines were cultured in Dulbecco's modified Eagle medium supplemented with $10 \%$ fetal bovine serum (FBS), $100 \mathrm{U} / \mathrm{ml}$ penicillin, and $100 \mu \mathrm{g} / \mathrm{ml}$ streptomycin in a $5 \% \mathrm{CO}_{2}$ humidified incubator.

Nuclear fractionation. To generate nuclear and cytoplasmic lysates, the Subcellular Protein Fractionation kit (Thermo Scientific, Rockford, IL, USA) was used. Stepwise lysis of cells generated both functional cytoplasmic and nuclear protein extracts. The protocol was performed according to the manufacturer's instructions.

Plasmid cDNA and small interfering RNA transfection. For EpICD plasmid cDNA transfection, EpICD cDNA (NCBI accession number: NM_002354.2; EpCAM cytoplasmic domain) was synthesized by CosmoGeneteck Co., Ltd. (Seoul, Korea) and inserted into the XhoI and BamHI sites of the pEGFP-NI vector (Clontech, Palo Alto, CA, USA). Transfection of EpICD plasmid DNA was performed using Lipofectamine 2000 transfection reagent (Invitrogen, Carlsbad, CA, USA) following the manufacturer's protocol. At $48 \mathrm{~h}$ after transfection, the cells were collected and used for further experiments.

Small interfering RNA (siRNA) sequences were employed to silence EpCAM expression. EpCAM siRNA and negative controls were purchased from Bioneer Corp. (Daejeon, Korea). Sequences for EpCAM-specific siRNA and negative control siRNA were as follows: EpCAM: sense 5'-GUGAG AACCUACUGGAUCA(dTdT)-3', antisense 5'-UGAUCCA GUAGGUUCUCAC(dTdT)-3', and negative control: sense 5'-CCUACGCCACCAAUUUCGU (dTdT)-3', antisense 5'-ACGAAAUUGGUGGCGUA GG(dTdT)-3'. Transfection of siRNA was performed with Lipofectamine RNAiMAX transfection reagent (Invitrogen) following the manufacturer's instructions.

Western blot analysis. Western blot was carried out in order to determine the effect of forced expression of EpICD and silencing EpCAM on the protein expression related to proliferation of the HCC cell lines. Cell lysates were resolved using a $10 \%$ polyacrylamide gel in a sodium dodecyl sulfate buffer and electrophoresis. After transfer onto a polyvinylidene difluoride membrane, the blots were incubated with anti-EpCAM (Calbiochem), EpICD (Abcam), active $\beta$-catenin (Merck Millipore, Billerica, MA, USA), c-myc (Abcam), cyclin D1 (Santa Cruz Biotechnology, Santa Cruz, CA, USA), $\beta$-catenin (BD Biosciences), and 

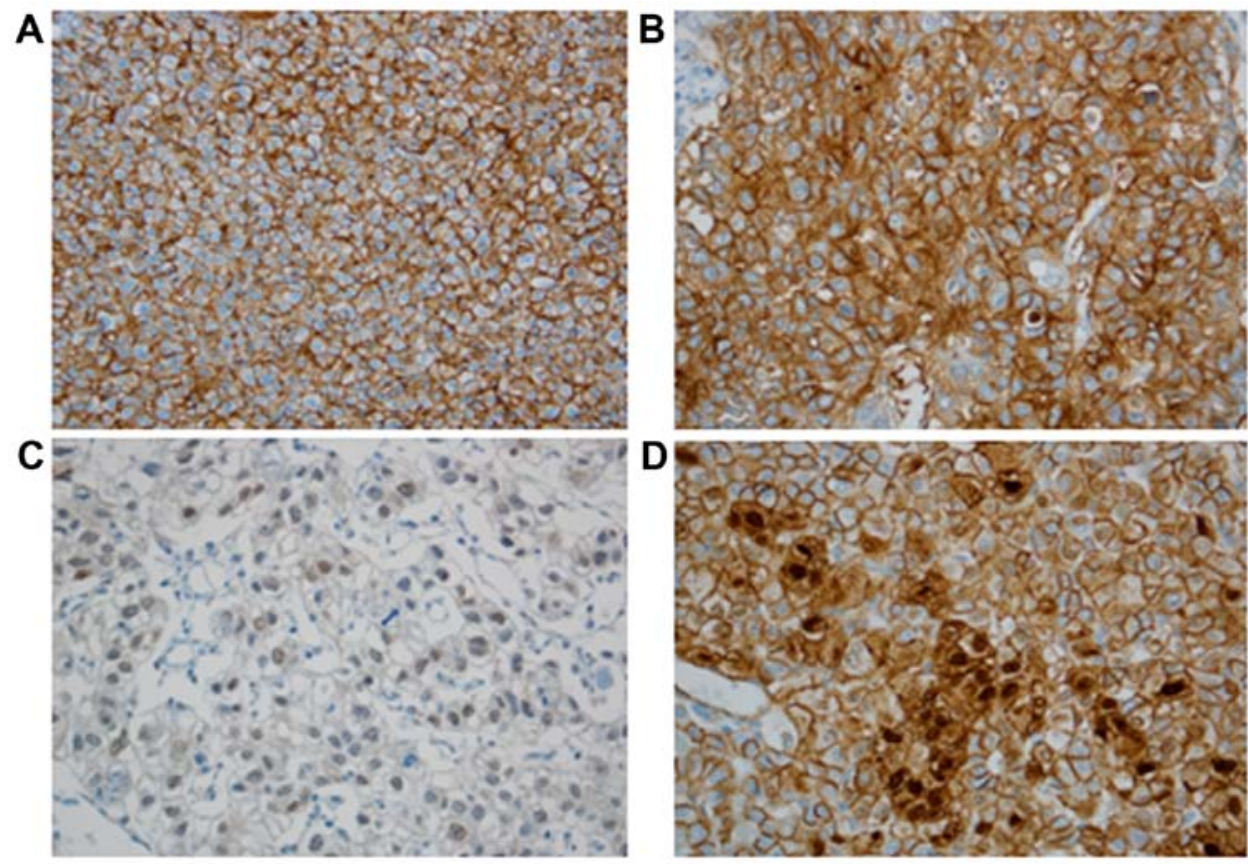

Figure 1. Immunohistochemical expression of EpEx, EpICD and $\beta$-catenin in hepatocellular carcinoma. (A) EpEx is predominantly expressed on membrane (x200). (B and C) EpICD is observed as membranous, cytoplasmic and nuclear immunostaining (x400). (D) $\beta$-catenin expressed on membrane, cytoplasm and nucleus (x400).

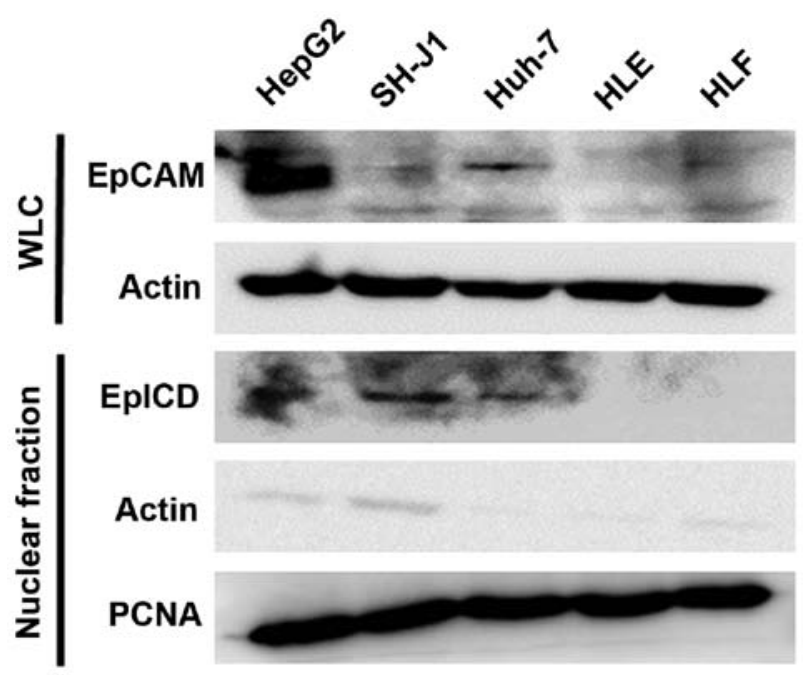

Figure 2. Western blot analysis of EpCAM and nuclear EpICD in whole cell lysate (WCL) and nuclear fraction from hepatocellular carcinoma cell lines. Actin and proliferating cell nuclear antigen (PCNA) were used as markers of the cytoplasmic and nuclear fractions, respectively. The expression level of EpCAM was higher in HepG2 and Huh-7 cell lines than in other cell lines. Nuclear translocation of EpICD was detected in the nuclear fraction of HepG2, SH-J1 and Huh-7 cell lines.

E-cadherin (BD Biosciences). The blots were developed using secondary antibody, and immune complexes were visualized using an enhanced chemiluminescence detection system (Amersham Biosciences, Buckinghamshire, UK). They were then analyzed with a LAS-3000 luminescent image analyzer (FujiFilm, Tokyo, Japan).

Cell proliferation assay. To evaluate the effect of EpICD gene transfection and silencing of EpCAM on the proliferation of
HCC cell lines, an MTT (3-(4,5-dimethylthiazol-2-yl)-2,5-diphenyltetrazolium bromide) assay was conducted. Briefly, the cells of each group were seeded into 96-well plates at 3000 cells per well. After 24 and $48 \mathrm{~h}$ of incubation, the MTT substrate (Sigma, St. Louis, MO, USA) was added to each well, and the cells were incubated at $37^{\circ} \mathrm{C}$ for $4 \mathrm{~h}$. Following elimination of the culture medium, the cells were dissolved in $0.2 \mathrm{ml}$ of dimethyl sulfoxide. The optical density was measured using a microplate reader (Bio-Rad, Hercules, CA, USA) at a wavelength of $560 \mathrm{~nm}$. Each experiment was repeated three times.

In vitro migration and invasion assays. The cell migration assay was performed using a 24-Transwell migration chamber (Corning Life Sciences, Acton, MA, USA), and the cell invasion assay was performed in a 24-Transwell BioCoat Matrigel invasion chamber (BD Biosciences) according to the manufacturer's instructions. For migration assay, $2 \times 10^{5} \mathrm{HepG} 2$ and HLE cells were seeded in serum-free medium in the upper chamber. For migration assay, $2 \times 10^{3}$ HepG2 and HLE cells were seeded. The cells, which either migrated to or invaded the lower surface of the membrane, were fixed with methanol and stained with a dye for $10 \mathrm{~min}$. Their numbers were counted from 10 random microscopic fields at x100 magnification.

Statistical analysis. Statistical analysis was performed using the Statistical Package for the Social Sciences (SPSS) and assessed using the Chi-square test and Student's t-test. Overall survival was calculated using the Kaplan-Meier method. Statistical significance was assumed at $\mathrm{p}<0.05$.

\section{Results}

EpEx, EpICD, and $\beta$-catenin expression in HCC and clinicopathologic correlations. EpEx expression was detected in 
Table I. Correlation between nuclear expression of EpICD or EpCAM and clinicopathological factors.

\begin{tabular}{|c|c|c|c|c|c|c|c|}
\hline \multirow[b]{2}{*}{ Factors } & \multirow[b]{2}{*}{ Total } & \multicolumn{3}{|c|}{ EpICD nuclear expression } & \multicolumn{3}{|c|}{ EpCAM } \\
\hline & & Negative & Positive & p-value & Negative & Positive & p-value \\
\hline \multicolumn{8}{|c|}{ Differentiation } \\
\hline G1-2 & 52 & $48(92.3)$ & $4(7.7)$ & 0.03 & $30(57.7)$ & $22(42.3)$ & 0.174 \\
\hline G3-4 & 48 & $33(68.8)$ & $15(31.2)$ & & $33(68.8)$ & $15(31.3)$ & \\
\hline \multicolumn{8}{|l|}{ Tumor size } \\
\hline$<5 \mathrm{~cm}$ & 65 & $55(84.6)$ & $10(14.4)$ & 0.161 & $41(63.1)$ & $24(36.9)$ & 0.575 \\
\hline$\geq 5 \mathrm{~cm}$ & 35 & $26(74.3)$ & $9(25.7)$ & & $22(62.9)$ & $13(37.1)$ & \\
\hline \multicolumn{8}{|c|}{$\mathrm{T}$ classification } \\
\hline $\mathrm{T} 1,2$ & 76 & $65(85.5)$ & $11(14.5)$ & 0.044 & $61(80.3)$ & $15(19.7)$ & 0.136 \\
\hline $\mathrm{T} 3,4$ & 24 & $16(66.7)$ & $8(33.3)$ & & $16(66.7)$ & $8(33.3)$ & \\
\hline \multicolumn{8}{|l|}{ Recurrence } \\
\hline Negative & 67 & $56(83.6)$ & $11(16.4)$ & 0.249 & $42(62.7)$ & $25(37.3)$ & 0.553 \\
\hline Positive & 33 & $25(75.8)$ & $8(24.2)$ & & $21(63.6)$ & $12(36.4)$ & \\
\hline \multicolumn{8}{|c|}{ Vascular invasion } \\
\hline Negative & 37 & $31(83.8)$ & $6(16.2)$ & 0.395 & $25(67.6)$ & $12(32.4)$ & 0.306 \\
\hline Positive & 63 & $50(79.4)$ & $13(20.6)$ & & $38(60.3)$ & $25(39.7)$ & \\
\hline \multicolumn{8}{|c|}{$\beta$-catenin nuclear expression } \\
\hline Negative & 90 & $76(84.4)$ & 14 (15.6) & 0.02 & $55(61.1)$ & 35 (38.9) & 0.207 \\
\hline Positive & 10 & $5(50)$ & $5(50)$ & & $8(80.0)$ & $2(20.0)$ & \\
\hline \multicolumn{8}{|l|}{ Ki-67 } \\
\hline Negative & 54 & $48(88.9)$ & $6(11.1)$ & 0.027 & $34(63.0)$ & $20(37.0)$ & 0.580 \\
\hline Positive & 46 & $33(71.7)$ & $13(28.3)$ & & $29(63.0)$ & $17(37.0)$ & \\
\hline \multicolumn{8}{|l|}{ E-cadherin } \\
\hline Loss & 22 & $11(50)$ & $11(50)$ & $<0.001$ & $16(72.7)$ & $6(27.3)$ & 0.208 \\
\hline Preserved & 78 & 70 (89.7) & $8(10.3)$ & & $47(60.3)$ & $31(39.7)$ & \\
\hline \multicolumn{8}{|l|}{ ЕрСАM } \\
\hline Negative & 77 & $61(79.2)$ & $16(20.8)$ & 0.309 & & & \\
\hline Positive & 23 & $20(87.0)$ & $3(13.0)$ & & & & \\
\hline
\end{tabular}

37 out of 100 HCC cases (37\%), showing predominantly membrane staining. EpICD immunoreactivity was observed as nuclear, cytoplasmic, and membranous immunostaining. Nuclear EpICD expression was seen in 19 of 100 (19\%) cases; cytoplasmic and membranous expression were observed in $49 \%(49 / 100)$ and $17 \%$ (17/100) of cases, respectively. No nuclear EpICD expression was present in non-neoplastic hepatocytes. Nuclear expression of $\beta$-catenin was noted in $10 \%$ (10/100) of HCC cases (Fig. 1).

The correlations between nuclear translocation of EpICD and clinicopathological variables are summarized in Table I. The nuclear translocation of EpICD was significantly higher in grades 3-4 (Edmondson-Steiner's grade) as compared with grades $1-2(\mathrm{p}=0.030)$. There was a significant correlation between the nuclear translocation of EpICD and a high $\mathrm{T}$ category (T1-2 vs. T3-4, $\mathrm{p}=0.044$ ). The nuclear translocation of EpICD was significantly correlated with the expression of nuclear $\beta$-catenin $(p=0.02)$, and Ki-67 $(p=0.027)$. Moreover, it was also correlated with a loss of E-cadherin expression $(\mathrm{p}<0.001)$. No significant correlation was found between the nuclear translocation of EpICD and other clinicopathological variables, including tumor size $(\mathrm{p}=0.161)$, tumor recurrence $(\mathrm{p}=0.249)$, vascular invasion $(\mathrm{p}=0.395)$, and EpCAM expression $(\mathrm{p}=0.309)$.

In addition, we also analyzed the correlation between EpCAM and clinicopathologic variables. However, none of the clinicopathologic variables had a statistically significant correlation with the EpCAM expression. The overall survival analysis showed no significant difference in survival based on the nuclear expression of EpICD $(\mathrm{p}=0.586)$.

EpCAM expression in HCC cell lines. The expression level of the EpCAM protein was higher in the HepG2 and Huh-7 cell lines than in other cell lines. To demonstrate the presence of EpICD in the nuclei, we performed a western blot analysis of the nuclear fraction of HCC cell lines. Nuclear translocation of EpICD was detected in the nuclear fraction of the HepG2, SH-J1, and Huh-7 cell lines (Fig. 2). 
A

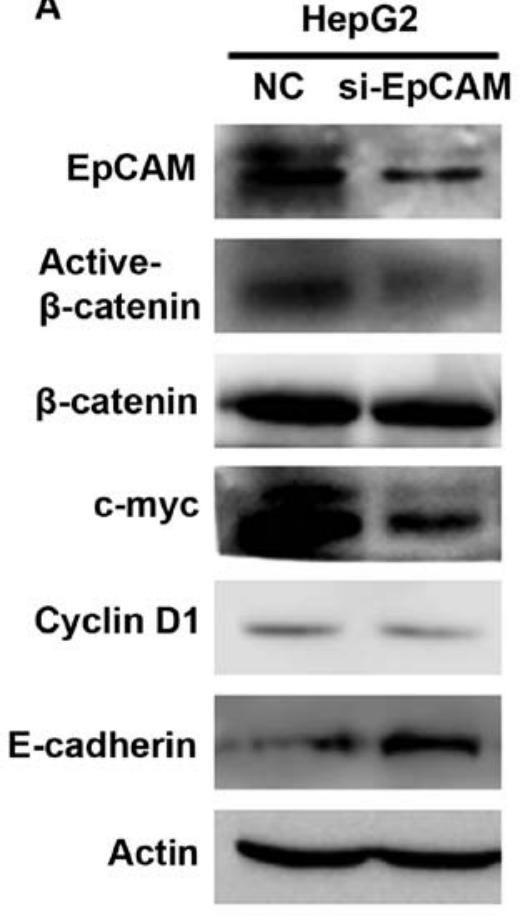

B

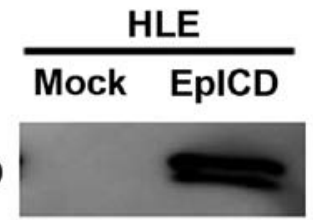

Active$\beta$-catenin

$\beta$-catenin

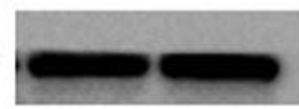

c-myc

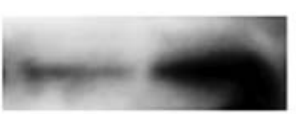

Cyclin D1

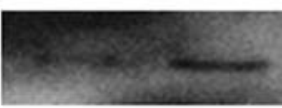

E-cadherin

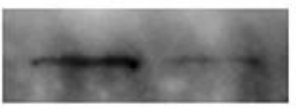

Actin

Figure 3. Downregulated EpCAM and overexpressed EpICD regulates the expression of active $\beta$-catenin, EpCAM target genes. Downregulation of EpCAM decreases the expression of the active form $\beta$-catenin, c-myc and cyclin D1. E-cadherin was significantly increased in the HepG2 cells (A). Overexpression of EpICD increases the expression of the active form $\beta$-catenin, c-myc and cyclin D1 in HLE cells, while the expression of E-cadherin was decreased (B).

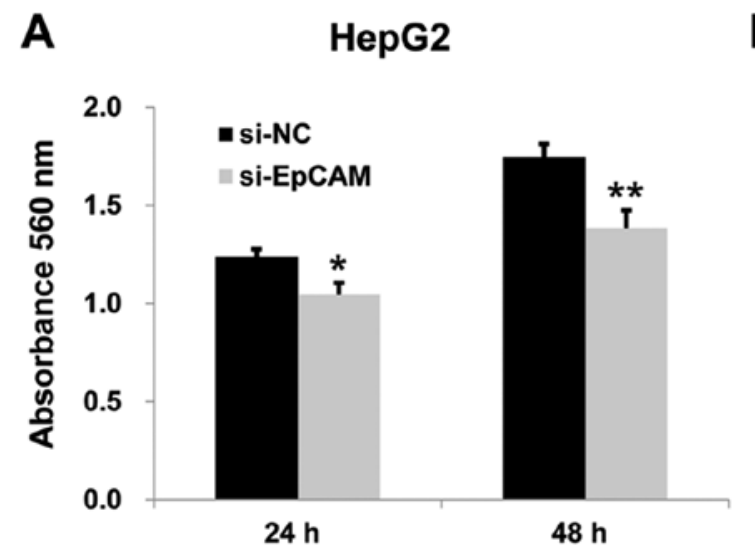

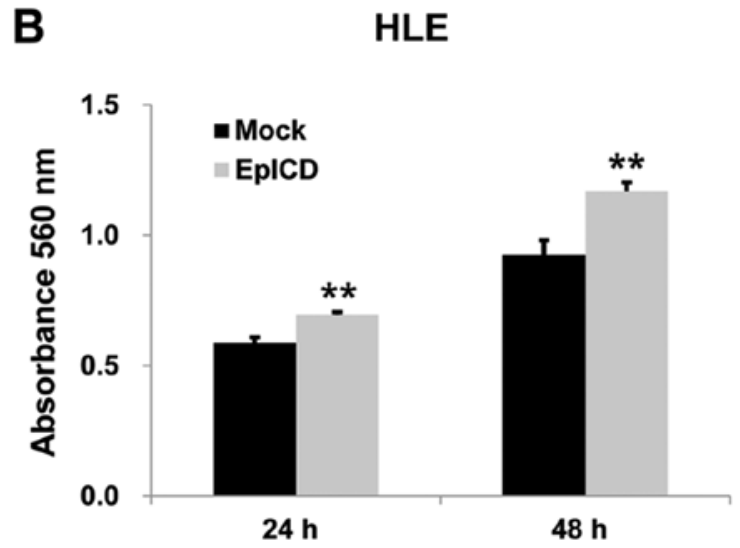

Figure 4. In vitro proliferation assay. Downregulation of EpCAM by siRNA decreased the cell proliferation when compared to that of the control (A). Proliferation of HLE cells that transfected with EpICD cDNA was significantly higher than that of the controls $(\mathrm{B})\left({ }^{*} \mathrm{p}<0.05,{ }^{* *} \mathrm{p}<0.01\right)$.

Influence of EpICD silencing and overexpression on expression of nuclear $\beta$-catenin, $c$-myc and cyclin D1. EpCAM siRNA was used to silence EpCAM, and the results showed that EpCAM siRNA lead to a marked decrease in EPCAM expression. Western blot analysis revealed that the downregulation of EpCAM decreased the expression of the active form $\beta$-catenin in HepG2 cells. Downregulation of EpCAM also induced a diminished expression level of EpCAM target genes, such as c-myc and cyclin D1. In contrast, E-cadherin was significantly increased in the EpCAM siRNA group compared to the controls (Fig. 3A).

To investigate the mechanism by which the overexpression of EpICD induces cell proliferation, the expression of nuclear $\beta$-catenin and EpCAM target genes was analyzed using western blot. The result revealed that the overexpression of EpICD increases the expression of the active form of $\beta$-catenin in HLE cells. In addition, c-myc and cyclin D1 were also highly expressed in the EpICD-transfected HLE cells, while the expression of E-cadherin was decreased (Fig. 3B).

Effect of EpICD silencing and overexpression on cell proliferation, migration, and invasion. To investigate the effect of silencing EpCAM on the proliferation of the HepG2 cell line, MTT assay was performed. After 24 and $48 \mathrm{~h}$, the absorbance of the silencing EpCAM group was significantly lower than those of the controls $(\mathrm{p}<0.05, \mathrm{p}<0.01$, Fig. 4A). Silencing EpCAM gene expression inhibited the migration and invasion capacity of both the HepG 2 and HLE cell lines. These results 

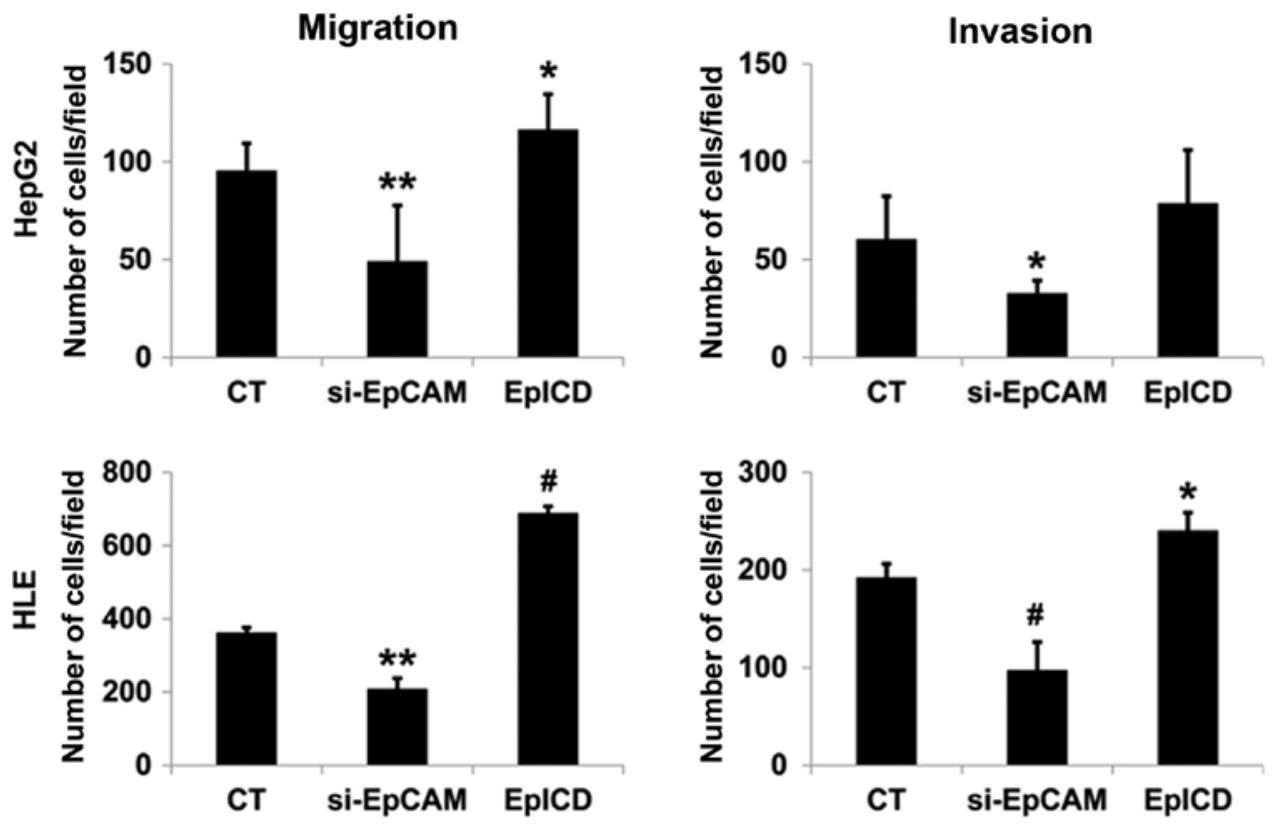

Figure 5. Migration and invasion assay. The migration and invasive potential were significantly reduced in EpCAM downregulated cell lines compared to control cells. Migration and invasion capacities increased with EpICD overexpression $\left({ }^{*} \mathrm{p}<0.05,{ }^{* *} \mathrm{p}<0.01,{ }^{*} \mathrm{p}<0.001\right)$.

indicated that the downregulation of EpCAM expression inhibited the proliferation, migration, and invasion of tumor cells (Fig. 5).

EpICD overexpression in HLE cells by EpICD cDNA resulted in significantly increased cell proliferation when compared to that of the control ( $<<0.01, \mathrm{p}<0.01$, Fig. 4B). Overexpression of EpICD in HLE cells leads to a significant increase in cell migration and invasion when compared to control cells ( $\mathrm{p}<0.001$, $\mathrm{p}<0.05$, Fig. 5).

\section{Discussion}

We determined the role of EpICD and the clinical significance of nuclear expression of EpICD in HCC. The main findings of this study were: i) nuclear expression of EpICD and $\beta$-catenin was detected in $19 \%$ and $10 \%$ of HCC patients, respectively; ii) nuclear expression of EpICD was significantly correlated with the nuclear expression of $\beta$-catenin, high tumor grade, high T category, and high Ki-67 index; iii) forced overexpression of EpICD in HCC cells increased the expression levels of the active form of $\beta$-catenin, c-myc and cyclin D1, whereas silencing EpCAM decreased the expression of the active form of $\beta$-catenin, c-myc, and cyclin D1; iv) EpICD overexpression increased cell proliferation, migration, and invasion. Silencing EpCAM gene expression inhibited proliferation, migration, and invasion in HCC cells.

EpCAM is a transmembrane glycoprotein that plays an important role in cell adhesion, proliferation, differentiation, migration, cell cycle regulation, and stem cell signaling (15). In HCC, EpCAM is known as a stemness marker, which is associated with aggressive behavior, chemoresistance, and poor prognosis (16). Although several studies have investigated the expression of the full length of EpCAM, the expression of EpICD and its role in tumorigenesis have not been studied in HCC.
EpCAM predominantly contributes to proliferation, invasion, and metastasis by regulating $\beta$-catenin signaling and E-cadherin mediated-adhesion (9,17). Maetzel et al (9) revealed that signaling by EpCAM requires regulated intramembrane proteolysis to release its intracellular domain EpICD. The sequential proteolysis of EpCAM by TACE and PS-2 produces EpEX and EpICD. The released EpICD forms a nuclear complex with $\beta$-catenin, Lef-1, and FHL2, and this transcription complex binds the DNA to activate the target genes (9). Expression of EpCAM is associated with upregulation of target genes, including $c$-myc and cyclins, which enhance proliferation $(18,19)$. We observed that overexpression of EpICD in HCC cells increased the expression of the active form of $\beta$-catenin, c-myc, and cyclin D1 and significantly increased the rate of cell proliferation. We found that nuclear EpICD expression was significantly correlated with a high Ki-67 proliferation index. These findings suggest that EpICD may be acting as an oncogenic signal transducer in HCC by activating $\beta$-catenin to promote proliferation.

EpCAM can inhibit E-cadherin-mediated cell-to-cell adhesion by disrupting the link between $\alpha$-catenin and F-actin (17). It is known that the cytoplasmic domain of EpCAM is required for its negative effect on cadherins (20). In addition, dissociation of cadherin adhesion leads to the accumulation of intracellular $\beta$-catenin and enhances cell proliferation (21). The results of this study are in agreement with these findings. Overexpression of EpICD decreased the expression of E-cadherin, whereas downregulation of EpCAM increased the expression of E-cadherin in HCC cells. The loss of E-cadherin function is a crucial step in epithelial-mesenchymal transition (EMT) (22). EMT is a biologic process in which polarized epithelial cells acquire the motile and invasive characteristics of mesenchymal cells, resulting in invasion and metastasis (22). EpICD regulates reprogramming and EMT genes, including c-Myc, Oct4, SOX2 and Nanog (21). Downregulation of EpICD 
suppresses the expression of EMT-related transcription factors Snail, Slug, Twist, and TCF4, thus reducing tumor invasiveness $(21,23)$. These transcriptional factors are well-known repressors of E-cadherin expression (24,25). Jachin et al (12) demonstrated that the nuclear expression of EpICD, which paralleled the nuclear expression of $\beta$-catenin, was increased in tumor buds in invasive front extrahepatic cholangiocarcinoma. Forced overexpression of EpICD enhanced the cell motility and invasiveness of cholangiocarcinoma cells (12). These findings were consistent with the results of our study in that forced EpICD overexpression increased migration and invasion, while silencing EpCAM expression suppressed migration and invasion in HCC cells.

Recently, there is increasing interest in the role of EpICD in cancer progression, aggressive behavior, and poor prognosis. Nuclear EpICD staining was observed in undifferentiated and poorly differentiated thyroid cancer, but not in well-differentiated thyroid cancer (10). It has also been demonstrated that the expression of nuclear EpICD is associated with a high tumor grade in colon cancer and extrahepatic cholangiocarcinoma $(12,21)$. Our results showed that the nuclear expression of EpICD was correlated with a high tumor grade and a high $\mathrm{T}$ category in HCC. This finding suggests that EpICD might be associated with HCC progression. There were no significant relationships between survival and nuclear EpICD expression in our study. However, nuclear EpICD expression has been correlated with a poor prognosis in thyroid and breast cancers and is significantly associated with tumor recurrence in breast cancer $(10,13)$. Further studies with a larger number of cases will be needed to confirm the prognostic significance of nuclear EpICD expression in $\mathrm{HCC}$.

EpCAM is a well-known therapeutic target antibody against epithelial tumors. The European Medicines Agency approved the use of catumaxomab (Removab ${ }^{\circledR}$ ), a trifunctional bispecific antibody targeting EpCAM, for the intraperitoneal treatment of malignant ascites (26). Clinical trials of EpCAM have already been performed in head and neck squamous cell carcinoma and bladder cancer patients $(27,28)$. A recent study attempted to verify the usefulness of EpCAM inhibitors for the treatment of HCC (29). Based on our results, EpCAM signaling pathway-associated RIP and EpICD can be considered a therapeutic target in HCC. Extensive further studies and verification will be necessary in order to confirm the potential of the EpICD inhibitor for use in the treatment of patients with HCC.

In conclusion, this study demonstrated an association between the nuclear expression of EpICD and $\beta$-catenin in HCC. The overexpression of EpICD in HCC cells induced a concomitant nuclear expression of the $\beta$-catenin and EpCAM target genes. The overexpression of EpICD significantly enhanced cell proliferation, migration, and invasion, while silenced EpCAM suppressed the proliferation, migration, and invasion in HCC cells in vitro. These findings support that the RIP-mediated EpCAM signaling pathway is involved in $\mathrm{HCC}$, and EpICD plays important roles in HCC progression by modulating the expression of target genes of EpCAM. Therefore, the RIP-mediated EpCAM signaling pathway, including EpICD, may be a possible candidate for molecular targeting in future treatments of HCC.

\section{Acknowledgements}

This work was supported by the National Research Foundation of Korea (NRF) grant funded by the Korean Government (MSIP) (no. 2008-0062279).

\section{References}

1. Ferlay J, Soerjomataram I, Dikshit R, Eser S, Mathers C, Rebelo M, Parkin DM, Forman D and Bray F: Cancer incidence and mortality worldwide: Sources, methods and major patterns in GLOBOCAN 2012. Int J Cancer 136: E359-E386, 2015.

2. Graf D, Vallbohmer D, Knoefel WT, Kröpil P, Antoch G, Sagir A and Häussinger D: Multimodal treatment of hepatocellular carcinoma. Eur J Intern Med 25: 430-437, 2014.

3. Litvinov SV, Velders MP, Bakker HA, Fleuren GJ and Warnaar SO: Ep-CAM: A human epithelial antigen is a homophilic cell-cell adhesion molecule. J Cell Biol 125: 437-446, 1994

4. Baeuerle PA and Gires O: EpCAM (CD326) finding its role in cancer. Br J Cancer 96: 417-423, 2007.

5. van der Gun BT, Melchers LJ, Ruiters MH, de Leij LF, McLaughlin PM and Rots MG: EpCAM in carcinogenesis: The good, the bad or the ugly. Carcinogenesis 31: 1913-1921, 2010.

6. Went PT, Lugli A, Meier S, Bundi M, Mirlacher M, Sauter G and Dirnhofer S: Frequent EpCam protein expression in human carcinomas. Hum Pathol 35: 122-128, 2004.

7. Schmidt M, Scheulen ME, Dittrich C, Obrist P, Marschner N, Dirix L, Schmidt M, Ruttinger D, Schuler M, Reinhardt C, et al: An open-label, randomized phase II study of adecatumumab, a fully human anti-EpCAM antibody, as monotherapy in patients with metastatic breast cancer. Ann Oncol 21: 275-282, 2010.

8. Niedzwiecki D, Bertagnolli MM, Warren RS, Compton CC, Kemeny NE, Benson AB, Eckhardt SG, Alberts S, Porjosh GN, Kerr DJ, et al: Documenting the natural history of patients with resected stage II adenocarcinoma of the colon after random assignment to adjuvant treatment with edrecolomab or observation: Results from CALGB 9581. J Clin Oncol 29: 3146-3152, 2011.

9. Maetzel D, Denzel S, Mack B, Canis M, Went P, Benk M, Kieu C, Papior P, Baeuerle PA, Munz M, et al: Nuclear signalling by tumour-associated antigen EpCAM. Nat Cell Biol 11: 162-171, 2009.

10. Ralhan R, Cao J, Lim T, Macmillan C, Freeman JL and Walfish PG: EpCAM nuclear localization identifies aggressive thyroid cancer and is a marker for poor prognosis. BMC Cancer 10: $331,2010$.

11. Ralhan R, He HC, So AK, Tripathi SC, Kumar M, Hasan MR, Kaur J, Kashat L, MacMillan C, Chauhan SS, et al: Nuclear and cytoplasmic accumulation of Ep-ICD is frequently detected in human epithelial cancers. PLoS One 5: e14130, 2010.

12. Jachin S, Bae JS, Sung JJ, Park HS, Jang KY, Chung MJ, Kim DG and Moon WS: The role of nuclear EpICD in extrahepatic cholangiocarcinoma: Association with $\beta$-catenin. Int $\mathrm{J}$ Oncol 45: 691-698, 2014.

13. Srivastava G, Assi J, Kashat L, Matta A, Chang M, Walfish PG and Ralhan R: Nuclear Ep-ICD accumulation predicts aggressive clinical course in early stage breast cancer patients. BMC Cancer 14: 726,2014

14. Kim DG, Park SY, Kim H, Chun YH, Moon WS and Park SH: A comprehensive karyotypic analysis on a newly established sarcomatoid hepatocellular carcinoma cell line $\mathrm{SH}-\mathrm{J} 1$ by comparative genomic hybridization and chromosome painting. Cancer Genet Cytogenet 132: 120-124, 2002.

15. Dollé L, Theise ND, Schmelzer E, Boulter L, Gires O and van Grunsven LA: EpCAM and the biology of hepatic stem/progenitor cells. Am J Physiol Gastrointest Liver Physiol 308: G233-G250, 2015.

16. Chan AW, Tong JH, Chan SL, Lai PB and To KF: Expression of stemness markers (CD133 and EpCAM) in prognostication of hepatocellular carcinoma. Histopathology 64: 935-950, 2014.

17. Winter MJ, Nagelkerken B, Mertens AE, Rees-Bakker HA, Briaire-de Bruijn IH and Litvinov SV: Expression of Ep-CAM shifts the state of cadherin-mediated adhesions from strong to weak. Exp Cell Res 285: 50-58, 2003.

18. Chaves-Pérez A, Mack B, Maetzel D, Kremling H, Eggert C, Harréus $U$ and Gires O: EpCAM regulates cell cycle progression via control of cyclin D1 expression. Oncogene 32: 641-650, 2013. 
19. Münz M, Kieu C, Mack B, Schmitt B, Zeidler R and Gires O: The carcinoma-associated antigen EpCAM upregulates c-myc and induces cell proliferation. Oncogene 23: 5748-5758, 2004.

20. Litvinov SV, Balzar M, Winter MJ, Bakker HA, Bruijn IH, Prins F, Fleuren GJ and Warnaar SO: Epithelial cell adhesion molecule (Ep-CAM) modulates cell-cell interactions mediated by classic cadherins. J Cell Biol 139: 1337-1348, 1997.

21. Lin CW, Liao MY, Lin WW, Wang YP, Lu TY and Wu HC: Epithelial cell adhesion molecule regulates tumor initiation and tumorigenesis via activating reprogramming factors and epithelial-mesenchymal transition gene expression in colon cancer. J Biol Chem 287: 39449-39459, 2012.

22. Kalluri R and Weinberg RA: The basics of epithelial-mesenchymal transition. J Clin Invest 119: 1420-1428, 2009.

23. Philip R, Heiler S, Mu W, Buchler MW, Zoller M and Thuma F: Claudin-7 promotes the epithelial-mesenchymal transition in human colorectal cancer. Oncotarget 6: 2046-2063, 2015.

24. Medici D, Hay ED and Olsen BR: Snail and Slug promote epithelial-mesenchymal transition through beta-catenin-T-cell factor-4-dependent expression of transforming growth factorbeta3. Mol Biol Cell 19: 4875-4887, 2008.
25. Garg M: Epithelial-mesenchymal transition - activating transcription factors - multifunctional regulators in cancer. World $\mathrm{J}$ Stem Cells 5: 188-195, 2013.

26. Bokemeyer C: Catumaxomab - trifunctional anti-EpCAM antibody used to treat malignant ascites. Expert Opin Biol Ther 10: 1259-1269, 2010.

27. MacDonald GC, Rasamoelisolo M, Entwistle J, Cuthbert W, Kowalski M, Spearman MA and Glover N: A phase I clinical study of intratumorally administered VB4-845, an anti-epithelial cell adhesion molecule recombinant fusion protein, in patients with squamous cell carcinoma of the head and neck. Med Oncol 26: 257-264, 2009.

28. Kowalski M, Guindon J, Brazas L, Moore C, Entwistle J, Cizeau J, Jewett MAS and MacDonald GC: A phase II study of oportuzumab monatox: An immunotoxin therapy for patients with noninvasive urothelial carcinoma in situ previously treated with bacillus Calmette-Guerin. J Urol 188: 1712-1718, 2012.

29. Ogawa K, Tanaka S, Matsumura S, Murakata A, Ban D, Ochiai T, Irie T, Kudo A, Nakamura N, Tanabe M, et al: EpCAM-targeted therapy for human hepatocellular carcinoma. Ann Surg Oncol 21: 1314-1322, 2014. 